University of Nebraska - Lincoln

DigitalCommons@University of Nebraska - Lincoln

Faculty Publications from Nebraska Center for Materials and Nanoscience, Nebraska Center Materials and Nanoscience

for (NCMN)

2009

\title{
Resistive hysteresis and interface charge coupling in BaTiO3-ZnO heterostructures
}

\author{
V. M. Voora \\ University of Nebraska-Lincoln \\ Tino Hofmann \\ University of Nebraska - Lincoln, thofmann4@unl.edu \\ M. Brandt \\ Universität Leipzig \\ M. Lorenz \\ Universität Leipzig \\ M. Grundmann \\ Universität Leipzig \\ See next page for additional authors
}

Follow this and additional works at: https://digitalcommons.unl.edu/cmrafacpub

Part of the Nanoscience and Nanotechnology Commons

Voora, V. M.; Hofmann, Tino; Brandt, M.; Lorenz, M.; Grundmann, M.; Ashkenov, N.; and Schubert, Mathias, "Resistive hysteresis and interface charge coupling in BaTiO3-ZnO heterostructures" (2009). Faculty Publications from Nebraska Center for Materials and Nanoscience. 102.

https://digitalcommons.unl.edu/cmrafacpub/102

This Article is brought to you for free and open access by the Materials and Nanoscience, Nebraska Center for (NCMN) at DigitalCommons@University of Nebraska - Lincoln. It has been accepted for inclusion in Faculty Publications from Nebraska Center for Materials and Nanoscience by an authorized administrator of DigitalCommons@University of Nebraska - Lincoln. 


\section{Authors}

V. M. Voora, Tino Hofmann, M. Brandt, M. Lorenz, M. Grundmann, N. Ashkenov, and Mathias Schubert 


\title{
Resistive hysteresis and interface charge coupling in $\mathrm{BaTiO}_{3}-\mathrm{ZnO}$ heterostructures
}

\author{
V. M. Voora, ${ }^{1, a)}$ T. Hofmann, $^{1}$ M. Brandt, ${ }^{2}$ M. Lorenz, ${ }^{2}$ M. Grundmann, ${ }^{2}$ N. Ashkenov, ${ }^{2}$ \\ and M. Schubert ${ }^{1}$ \\ ${ }_{1}^{1}$ Department of Electrical Engineering, Nebraska Center for Materials and Nanoscience, \\ University of Nebraska-Lincoln, Lincoln, Nebraska 68588, USA \\ ${ }^{2}$ Institut für Experimentelle Physik II, Universität Leipzig, 04103 Leipzig, Germany
}

(Received 23 February 2009; accepted 18 March 2009; published online 7 April 2009; publisher error corrected 13 May 2009)

\begin{abstract}
We report on temperature, time, and voltage dependent resistive hysteresis measurements of $\mathrm{BaTiO}_{3}-\mathrm{ZnO}$ heterostructures grown on (001) $\mathrm{Si}$ substrates by pulsed laser deposition. We observe a diodelike behavior and cycling-voltage dependent hysteresis formation under forward bias. We explain these effects with depletion layer formation between the $\mathrm{ZnO}$ and $\mathrm{BaTiO}_{3}$ layers, an additional barrier due to the spontaneous polarization of $\mathrm{ZnO}$ and the ferroelectric nature of $\mathrm{BaTiO}_{3}$. The disappearance of the resistive hysteresis above the ferroelectric-paraelectric phase transition temperature of $\mathrm{BaTiO}_{3}$ conformed that the hysteresis is related to the ferroelectricity of $\mathrm{BaTiO}_{3}$. Time dependent resistance measurements reveal memory effects. (C) 2009 American Institute of Physics. [DOI: 10.1063/1.3116122]
\end{abstract}

Device integration approaches scaling dimensions where quantum principles may dictate incorporation of resistive instead of charge switching concepts. Future electronic memory concepts may incorporate resistance change upon external stimuli, either within a material or across a heterostructure interface. Bistable, switchable states of resistivity have been reported for organic and inorganic materials and heterostructures, including ferroelectric perovskite-structure materials. ${ }^{1-4}$ Understanding of the intrinsic physical mechanisms of reported resistance switching is not exhaustive. In this paper we report on temperature, time, and voltage dependent resistive hysteresis properties across a $\mathrm{BaTiO}_{3}(\mathrm{BTO})-\mathrm{ZnO}$ heterostructure. We identify a physical control mechanism-the coupling between spontaneous interface charge of the wurtzite-structure $\mathrm{ZnO}$ layer and the perovskite-structure BTO layer (interface charge coupling). This coupling provides a permanent offset (bias) for the external stimuli (voltage) under which the actual resistance switch occurs. This asymmetry may find use for astable, bistable, or multistable resistance device operation mode.

The room temperature perovskite-structure phase of BTO possesses electrically reversible spontaneous polarization and is well investigated due to its piezoelectric, pyroelectric, and ferroelectric properties. ${ }^{5-7} \mathrm{ZnO}$ received substantial attention due to its wide spectral transparency and the strongly excitonic, direct fundamental electronic band-toband transition in the ultraviolet region, ${ }^{8}$ for example. Under typical growth conditions $\mathrm{ZnO}$ crystallizes in the piezoelectric wurtzite-structure phase, and therefore possesses an irreversible spontaneous polarization $P_{\mathrm{sz}}$. The spontaneous polarization causes a permanent internal electric field. Heterostructures composed of $\mathrm{ZnO}$ and BTO thin films are interesting because of the anticipated coupling effects between the electrically switchable spontaneous polarization of BTO and the nonswitchable spontaneous polarization of $\mathrm{ZnO}^{9}{ }^{9}$ The nonswitchable spontaneous polarization of $\mathrm{ZnO}$

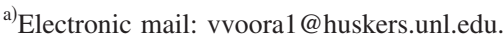

biases and thereby influences the switching behavior of the ferroelectric polarization charge in the adjacent BTO layer. ${ }^{9-13}$

Electro-optic Raman scattering revealed that the BTO layer possesses its ferroelectric perovskite structure at room temperature, and the disappearance of the distinctive perovskite structure mode above the Curie temperature indicated pinning of the perovskite lattice structure by the spontaneous $\mathrm{ZnO}$ interface polarization charge. ${ }^{10}$ Electric Sawyer-Tower (S-T) polarization (capacitance circuit) measurements indicated a strongly asymmetric and rectifying behavior of the heterostructure. ${ }^{12}$ Electro-optic ellipsometry investigations were indicative for internal rearrangement of free charge carriers near the BTO-ZnO interface upon external voltage cycling, giving rise to index-of-refraction hysteresis resembling the previously observed polarization (capacitance) hysteresis. ${ }^{11}$ The S-T measurements detect the charging and discharging ability (the history of electric polarization versus applied voltage) of a capacitive sample compared to a standard ideal capacitor. We developed a dielectric continuum model that describes the asymmetric polarization hysteresis behavior and thereby confirmed the influence of the spontaneous polarization of $\mathrm{ZnO}$ on the $\mathrm{BTO}$ switchable ferroelectric spontaneous polarization. ${ }^{13}$ Our model revealed the existence of a barrier between the $\mathrm{ZnO}$ and BTO interface, the formation of a depletion layer within the $\mathrm{ZnO}$ layer at the interface, and predicted as a fact that interface charge coupling between the $\mathrm{ZnO}$ and BTO layers offset (bias) the switching points (voltages) of the ferroelectric BTO polarization. The model predicted further a diodelike rectifying behavior, where in "forward" direction electrons can freely move within the proposed $n$-type $\mathrm{ZnO}$ layer and in "reverse" direction electrons are drained from the $\mathrm{ZnO}$ layer leaving behind a space charge region of width $w$ at the BTO-ZnO interface. This region gradually changes the capacitance of the sample into a series capacitance, controlled by the width $w$. Likewise, the resistance across the heterostructure should increase due to the increased depletion width with the elec- 


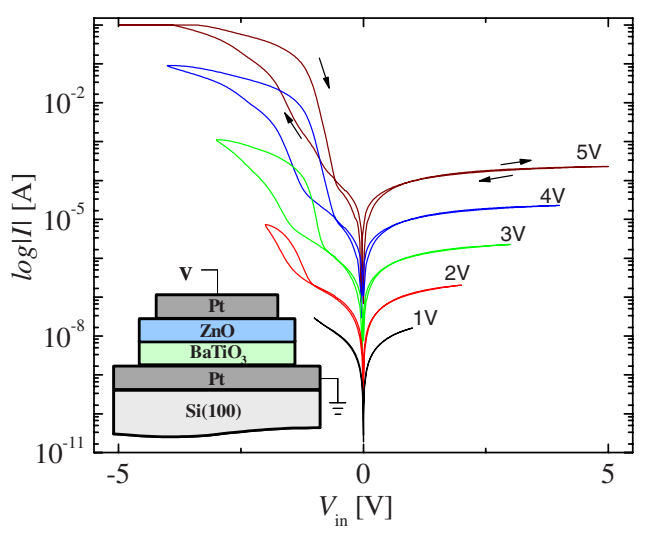

FIG. 1. (Color online) $I-V$ hysteresis characteristics of a $\mathrm{Pt} / \mathrm{BTO} / \mathrm{ZnO} / \mathrm{Pt}$ heterojunction. The bias voltage was swept as $-V_{\max } \rightarrow+V_{\max } \rightarrow-V_{\max }$. Arrows indicate the direction of the sweep. The $V_{\max }$ is $1,2,3,4$, and $5 \mathrm{~V}$. For clarity each $I-V$ curve, starting from $2 \mathrm{~V}$ is shifted successively by one order of magnitude. The inset shows the schematic of $\mathrm{Pt} / \mathrm{BTO} / \mathrm{ZnO} / \mathrm{Pt}$ heterostructure.

tric potential $V$ mostly dropping across the depleted region of the $\mathrm{ZnO}$ layer. The space charge region is further influenced by the spontaneous interface polarization. We identified voltages in forward and reverse direction at which the depletion layer occurs or disappears. ${ }^{13}$ These voltages reveal the formation of the ferroelectric spontaneous polarization biased by the $\mathrm{ZnO}$ spontaneous polarization. From this model we calculated the amount of the spontaneous polarization of $\mathrm{ZnO}$ as $P_{\mathrm{sz}}=-4.1 \mu \mathrm{C} / \mathrm{cm}^{2}$, which showed to be in good agreement with previous theoretical predictions. ${ }^{14}$ In this paper we report on temperature, time, and voltage dependent resistive (current-voltage) measurements in our BTO-ZnO heterostructure, and find that the observed behavior matches the scenario of the proposed interface charge coupling in BTO-ZnO heterostructures.

The BTO and ZnO layers were deposited by pulsed laser deposition, successively without breaking the vacuum, at $700{ }^{\circ} \mathrm{C}$ and 0.06 mbar substrate temperature and background oxygen partial pressure, respectively. The number of laser pulses for BTO was $10^{5}$, and for $\mathrm{ZnO} 2 \times 10^{4}$. A pulse energy of $600 \mathrm{~mJ}$ was used. The bottom and top metal electrodes were grown by sputtering using Pt forming Ohmic contacts. The thickness values of $d_{f}=1.45 \mu \mathrm{m}$ for BTO and $d_{z}$ $=0.5 \mu \mathrm{m}$ for $\mathrm{ZnO}$ were determined by ellipsometry, $\mathrm{x}$-ray, and transmission electron microscopy investigations. Structural (x-ray, electron microscopy), ${ }^{9}$ vibrational (Raman), ${ }^{10}$ electrical (polarization hysteresis), ${ }^{12}$ electro-optic (index-of refraction) hysteresis, ${ }^{11}$ and dielectric continuum polarization model (S-T response) ${ }^{13}$ analysis were performed as described previously. The BTO and $\mathrm{ZnO}$ layers were highly textured polycrystalline [BTO with (111) and $\mathrm{ZnO}$ with (0001) texture]. Electrical Hall-effect, infrared ellipsometry, ${ }^{8}$ and our dielectric continuum model analysis ${ }^{13}$ obtained that the $\mathrm{ZnO}$ layer is $n$-type conductive, with free electron concentration $N_{c} \approx 5.5 \times 10^{16} \mathrm{~cm}^{-3}$, while the BTO layer is highly resistive. The current-voltage $(I-V)$ measurements were recorded at different sweeping (maximum) voltages $\left(V_{\max }\right)$. In order to avoid irreversible damage to our sample we limited $V_{\max }$ to $5 \mathrm{~V}$. The current compliance was set to $1 \mathrm{~mA}$ corresponding to a pad size of $0.07 \mathrm{~cm}^{2}$. The sweeping direction of the applied bias voltage was $-V_{\max } \rightarrow+V_{\max } \rightarrow-V_{\max }$.

Figure 1 depicts $\log |I|-V$ curves obtained for $V_{\max }=1,2$,
3, 4, and $5 \mathrm{~V}$. For clarity subsequent $\log |I|-V$ curves are shifted by one order of current magnitude. The inset of the Fig. 1 shows the schematic of the $\mathrm{Pt} / \mathrm{BTO} / \mathrm{ZnO} / \mathrm{Pt}$ heterostructure. The heterostructure exhibits a diodelike current characteristics with high resistive behavior for positive voltages and conductive behavior for negative voltages. At $V_{\max }=1 \mathrm{~V}$, the $\log |I|-V$ curve is slightly asymmetric with higher resistance at positive voltages, and has no hysteresis. All the other $\log |I|-V$ loops show strong asymmetry and hysteresis behavior. The hysteresis occurs on the negative voltage side and sets in at approximately $-1.1 \mathrm{~V}\left(V_{\max }=2 \mathrm{~V}\right)$, $-0.8 \mathrm{~V}\left(V_{\max }=3 \mathrm{~V}\right)$, and $-0.6 \mathrm{~V}\left(V_{\max }=4 \mathrm{~V}, V_{\max }=5 \mathrm{~V}\right)$. The resistance drops during the downward sweep at approximately $-1.4 \mathrm{~V}$, and hysteresis increases with increasing $V_{\max }$. Complete saturation of the hysteresis may not have been reached here at $V_{\max }=5 \mathrm{~V}$, but those at $V_{\max }=4 \mathrm{~V}$ and $V_{\max }=5 \mathrm{~V}$ seem very similar indicating that saturation may be almost reached. Note that the loop at $V_{\max }=5 \mathrm{~V}$ is pinned due to the set current compliance limit.

The resistance switch during the downward sweep at approximately $-1.4 \mathrm{~V}$ points to the influence of the spontaneous interface polarization charge coupling of $\mathrm{ZnO}$ and BTO layers. This value matches with the previously observed voltage shift in electric polarization loops obtained by S-T polarization measurements of our sample. ${ }^{13}$ For small voltage loops $\left(V_{\max }=1 \mathrm{~V}\right)$ the $\log |I|-V$ resembles the varying resistance due to slight variation in the $\mathrm{ZnO}$ depletion layer at the BTO-ZnO interface. Due to the $\mathrm{ZnO}$ spontaneous interface charge, this depletion layer exists already at zero negative bias, and does not disappear for small negative bias. Once the bias reaches below approximately $-1.4 \mathrm{~V}$ (loop with $V_{\max }=2 \mathrm{~V}$ and higher), the spontaneous $\mathrm{ZnO}$ polarization is compensated and the depletion layer disappears. Then electric potential can build up across the BTO layer (the $\mathrm{ZnO}$ layer is seen idealistically as being conductive), and the ferroelectric polarization begins to build up. Both the flow of external charge compensating for the internal polarization development and the disappearance of the series resistance of the $\mathrm{ZnO}$ depletion layer account for the increase in conductivity, while the high resistance of the BTO layer limits the transport. At $V_{\max }=5 \mathrm{~V}$ the current density is four orders of magnitude smaller for positive bias than for negative bias voltages. With increasing negative bias voltage, the ferroelectric polarization of the BTO increases and reaches the maximum value when the voltage reaches its maximum value of $-V_{\max }$. As the bias voltage decreases from $-V_{\max }$, the orientation of the polarization of BTO would ideally remain until external bias and spontaneous interface charge compensate the BTO polarization and reversal of the latter would occur. This would lead to a square hysteresis loop, and our previous model analysis suggests this would occur close to zero bias. However, the BTO polarization occurs in multiple domains, and as the external voltage sweep is increasing from negative maximum voltage, nonideal depolarization processes of the BTO ferroelectric domains result in current decrease prior to the actual switching point. This causes the slight shift in the hysteresis onset toward smaller voltages for larger sweeping voltages.

Temperature-dependent $I-V$ measurements shown in Fig. 2 identify the ferroelectric nature of BTO as the major origin of the resistance hysteresis, while potentially existing defects and charges trapped at their sites cannot be fully ruled 


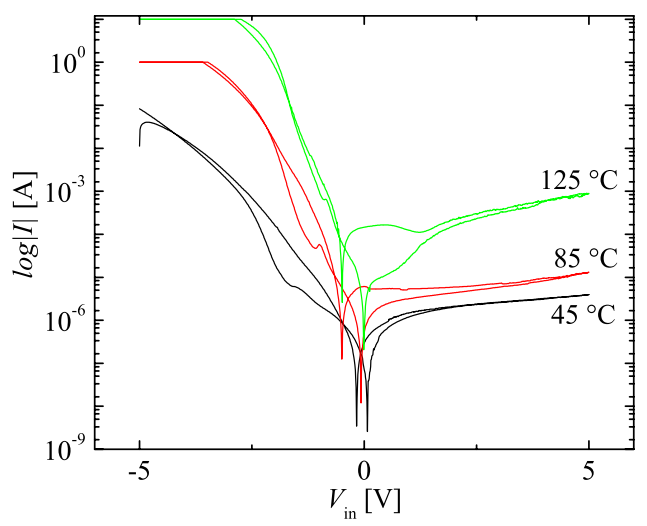

FIG. 2. (Color online) The $I-V$ loops of our BTO/ZnO heterostructure at different temperatures. For clarity, the curve at $85{ }^{\circ} \mathrm{C}\left(125^{\circ} \mathrm{C}\right)$ is shifted upward by one (two) order of magnitude.

out. ${ }^{1,15,16}$ At $125^{\circ} \mathrm{C}$ the hysteresis disappeared while the diodelike behavior is persistent. A small (logarithmic scale) hysteresis evolves for small bias voltages, the origin of which is unclear at this point. The disappearance of the hysteresis above the Curie temperature of BTO was also corroborated by our previous findings where at that temperature also the perovskite-structure lattice mode indicative for ferroelectric BTO disappeared in electro-optic Raman investigations. ${ }^{10}$ The latter proved transition of the BTO layer from its tetragonal ferroelectric to its cubic paraelectric phase in our sample. The higher temperature curves appear characteristic of transport supported by traps and defect charges. Temperature-dependent measurements of the recovery rate are underway, and will be discussed elsewhere.

We have further studied the time dependence of the observed resistance hysteresis in order to test their usability for potential information storage upon variation of external stimuli parameters. The top panel of the Fig. 3 presents time dependent current measurements at steady voltages. Included is also the time-dependent course of the external bias volt-

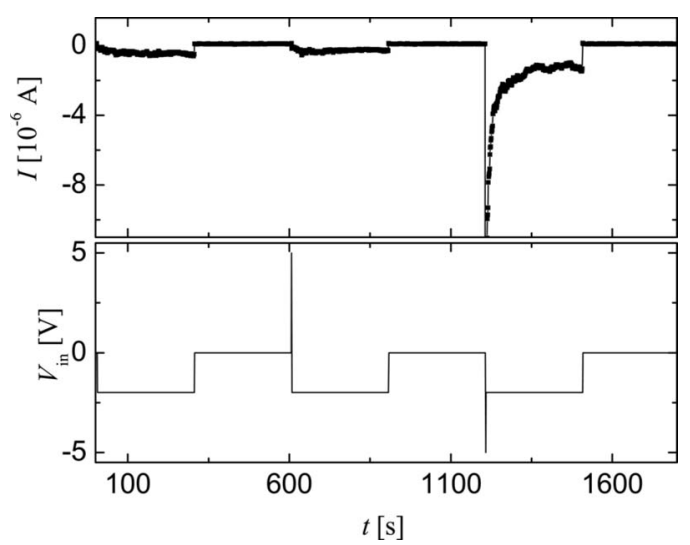

FIG. 3. The top panel of the figure shows the leakage current measurements as a function of time and applied voltage. The lower panel of the figure shows the voltage pulse sequence. The -2 and $0 \mathrm{~V}$ pulses were applied for $5 \mathrm{~min}$, and the 5 and $-5 \mathrm{~V}$ pulse were applied for one second each. age, which was held constant within and outside the hysteresis loop in Fig. $1(-2 \mathrm{~V}, 0 \mathrm{~V})$ and ramped to $+5 \mathrm{~V}$ and $-5 \mathrm{~V}$ for short periods of time. The bias of $-2 \mathrm{~V}$ can be regarded as read bias. The 0 and $2 \mathrm{~V}$ pulse were applied for the duration of $5 \mathrm{~min}$, and 5 and $-5 \mathrm{~V}$ pulses were applied for $1 \mathrm{~s}$. There is no significant change in the current at read bias after applying $\mathrm{a}+5 \mathrm{~V}$ short pulse. On the other hand the effect of $-5 \mathrm{~V}$ short pulse at read bias is significant. The structure immediately responds into a low resistance state after applying the large negative voltage pulse, and which stabilizes after approximately $1 \mathrm{~min}$. The low resistance is then approximately four times smaller than the high resistance.

We conclude that voltage dependent resistive hysteresis in BTO-ZnO heterostructures may enable resistance parameter write-read information storage devices. Time-dependent measurements of recyclability of the read-write process are currently underway and results will be reported elsewhere. We identify and discuss the interface charge coupling phenomenon in piezoelectric heterostructures, which may be employed for permanently biasing resistance switching voltages in such device structures.

We acknowledge support by the Deutsche Forschungsgemeinschaft (DFG) within the Sonderforschunsgbereich "Functional Oxide Interfaces" (DFG Sonderforschungsbereich 762 "Funktionalität Oxidischer Grenzflächen"), the $\mathrm{CoE}$ at UNL, the J. A. Woollam Foundation, and NSF MRSEC QSPIN (Grant No. DMR-0820521).

${ }^{1}$ G. Z. Liu, K. J. Jin, J. Qiu, M. He, H. B. Lu, J. Xing, Y. L. Zhou, and G. Z. Yang, Appl. Phys. Lett. 91, 252110 (2007).

${ }^{2}$ M. P. Singh, L. Mechin, W. Prellier, and M. Maglione, Appl. Phys. Lett. 89, 202906 (2006).

${ }^{3}$ J. Rodriguez Contreras, H. Kohlstedt, U. Poppe, R. Waser, C. Buchal, and N. A. Pertsev, Appl. Phys. Lett. 83, 4595 (2003).

${ }^{4}$ R. Oligschlaeger, R. Waser, R. Meyer, S. Karthäuser, and R. Dittmann, Appl. Phys. Lett. 88, 042901 (2006).

${ }^{5}$ C. R. Bowen, J. Gittings, I. G. Turner, F. Baxter, and J. B. Chaudhuri, Appl. Phys. Lett. 89, 132906 (2006).

${ }^{6}$ K. Ishikawa and T. Uemori, Phys. Rev. B 60, 11841 (1999).

${ }^{7}$ W. Zhong, D. Vanderbilt, and K. M. Rabe, Phys. Rev. B 52, 6301 (1995).

${ }^{8}$ C. Bundesmann, R. Schmidt-Grund, and M. Schubert, Springer Ser. Mater. Sci. 104, 79 (2008).

${ }^{9}$ M. Schubert, N. Ashkenov, T. Hofmann, H. Hochmuth, M. Lorenz, M. Grundmann, and G. Wagner, Ann. Phys. 13, 61 (2004).

${ }^{10}$ B. N. Mbenkum, N. Ashkenov, M. Schubert, M. Lorenz, H. Hochmuth, D. Michel, M. Grundmann, and G. Wagner, Appl. Phys. Lett. 86, 091904 (2005).

${ }^{11}$ V. M. Voora, T. Hofmann, M. Brandt, M. Lorenz, M. Grundmann, and M. Schubert, Phys. Status Solidi C 5, 1328 (2008).

${ }^{12}$ N. Ashkenov, M. Schubert, E. Twerdowski, H. V. Wenckstern, B. N. Mbenkum, H. Hochmuth, M. Lorenz, W. Grill, and M. Grundmann, Thin Solid Films 486, 153 (2005).

${ }^{13}$ V. M. Voora, T. Hofmann, M. Brandt, M. Lorenz, M. Grundmann, N. Ashkenov, and M. Schubert, J. Electron. Mater. 37, 1029 (2008).

${ }^{14}$ F. Bernardini and V. Fiorentini, Phys. Rev. B 56, R10024 (1997).

${ }^{15}$ P. W. M. Blom, R. M. Wolf, J. F. M. Cillessen, and M. P. C. M. Krijn, Phys. Rev. Lett. 73, 2107 (1994).

${ }^{16}$ D. J. Fu, J. C. Lee, S. W. Choi, S. J. Lee, T. W. Kang, M. S. Jang, H. I. Lee, and Y. D. Woo, Appl. Phys. Lett. 81, 5207 (2002). 